Revue interdisciplinaire des études canadiennes en

France

79 | 2015

Regards croisés Canada-Europe

\title{
Les impacts potentiels de l'AECG sur les secteurs financiers du Canada et de l'Union européenne
}

Potential impact of CETA on financial services in Canada and in the EU

Sébastien Labrecque et Nassiba Idebdou

\section{OpenEdition}

Journals

Édition électronique

URL : http://journals.openedition.org/eccs/558

DOI : $10.4000 /$ eccs.558

ISSN : 2429-4667

Éditeur

Association française des études canadiennes (AFEC)

Édition imprimée

Date de publication : 1 décembre 2015

Pagination : 7-28

ISSN : 0153-1700

Référence électronique

Sébastien Labrecque et Nassiba Idebdou, « Les impacts potentiels de l'AECG sur les secteurs

financiers du Canada et de l'Union européenne », Études canadiennes / Canadian Studies [En ligne],

79 | 2015, mis en ligne le 01 décembre 2016, consulté le 19 avril 2019. URL : http://

journals.openedition.org/eccs/558; DOI : 10.4000/eccs.558 


\title{
LES IMPACTS POTENTIELS DE L'AECG SUR LES SECTEURS FINANCIERS DU CANADA ET DE L'UNION EUROPÉENNE
}

\author{
Sébastien LABRECQUE ${ }^{1}$ et Nassiba IDEBDOU ${ }^{2}$ \\ Université d'Ottawa
}

L'Accord économique et commercial global (AECG) entre le Canada et l'Union européenne (UE) constitue un projet ambitieux qui facilitera l'accès à de nombreuses opportunités d'investissements transatlantiques et ce dans plusieurs secteurs. Cet article analyse spécifiquement les impacts potentiels du chapitre 15 sur les services financiers de l'AECG, en s'intéressant notamment aux défis que cet Accord posera aux institutions financières européennes voulant s'installer au Canada (et vice versa) et à l'enjeu d'une convergence règlementaire potentielle entre le Canada et l'UE en matière de règlementation financière en vertu de l'AECG.

The Comprehensive Economic Trade Agreement (CETA) involving Canada and the European Union (EU) is an ambitious project that aims to facilitate access to numerous transatlantic investing opportunities in multiple industries. This article specifically analyzes the potential implications of chapter 15 on financial services of CETA by looking for instance at challenges posed by the Agreement to financial institutions relocating to Canada (and vice versa) and at the issue of a potential regulatory convergence between Canada and the EU for financial regulations as a result of CETA.

Finalement conclu en septembre 2014, l'Accord économique et commercial global (AECG) entre le Canada et l'Union européenne (UE) vise à faciliter l'accès à de nombreuses opportunités d'investissements transatlantiques, et ce, dans une multitude de domaines. L’AECG est généralement décrit par le gouvernement canadien ainsi que par les médias comme le plus ambitieux et extensif accord commercial de l'histoire du Canada, plus vaste encore que l'Accord de libre-échange nord-américain (ALÉNA). Les services financiers constituent l'un des secteurs visés par l'AECG (cf chapitre 15 de l'AECG).

Dans une ère post crise économique où les règlementations financières ont été renforcées partout sur la planète, cet article pose la problématique de recherche suivante : quels seront les impacts potentiels du chapitre 15 de l'AECG sur les secteurs financiers du Canada et de l'Union européenne et sur la règlementation de ceux-ci ? Cette recherche se base sur la notion de convergence règlementaire pour tenter de répondre à notre problématique. À la lumière de la littérature théorique et empirique sur la convergence règlementaire, des similitudes entre

\footnotetext{
${ }^{1}$ Candidat au doctorat, École d'études politique, Université d'Ottawa

${ }^{2}$ Maîtrise ès arts, Affaires publiques et internationales, École supérieure d'affaires publiques et internationales, Université d'Ottawa.
} 


\section{Nassiba IDEBDOU et Sébastien LABRECQUE}

le chapitre 15 de l'AECG et l'Accord général sur le commerce des services (AGCS) et la possibilité d'adopter des exceptions prudentielles, nous avançons la thèse que l'AECG ne devrait pas mener à une convergence règlementaire majeure entre le Canada et l'UE en matière de services financiers. En ce sens, l'impact du chapitre 15 sur les secteurs financiers des deux blocs devrait être limité à court et moyen terme.

Pour développer cette thèse et y répondre, cet article propose un bref survol de l'AECG et dresse un portrait des secteurs financiers du Canada et de l'UE ainsi que de la règlementation de ces derniers. Enfin, la dernière section porte précisément sur le chapitre 15 et s'attarde aux bénéfices et aux coûts de la convergence règlementaire, aux exceptions prudentielles permises par l'AECG et au Comité des services financiers mis en place par l'Accord.

\section{1 - L'AECG en bref}

La relation commerciale entre le Canada et l'UE a toujours été historiquement très asymétrique. En effet, l'UE est le deuxième partenaire commercial du Canada derrière les États-Unis, tandis que le Canada se classe seulement au douzième rang des partenaires commerciaux de l'UE en excluant le commerce des services, catégorie où il arrive huitième (DEBLOCK ET Rioux 2011: 49) ${ }^{3}$. Il importe toutefois de souligner que le Canada se classe plus haut en terme d'investissements en figurant habituellement parmi les quatre premiers partenaires de l'Europe ( $3^{\mathrm{e}}$ ou $4^{\mathrm{e}}$ selon les données et les années) (DEBLOCK ET RIOUX 2011: 49, DUCHESNE ET MORIN 2013: 14). Tout comme pour le commerce, l'UE occupe le deuxième rang au niveau des investissements au Canada.

Les premières démarches formelles de la part du Canada pour renforcer le commerce et l'investissement avec l'Europe remontent à la fin $\mathrm{du} \mathrm{XX}^{\mathrm{e}}$ siècle. En 1997, l'UE a conclu une entente avec le Canada pour approfondir la coopération administrative au niveau des services douaniers et ainsi faciliter le commerce ${ }^{4}$. Les premières négociations de libre-échange menées par Ottawa avec un groupe de pays européens, l'Association européenne de libre-échange,

\footnotetext{
${ }^{3}$ The Canadian Trade Commissioner Service Foreign Affairs (2015), «Focus on the European Union », modifié le 13 mars 2015.

http://www.tradecommissioner.gc.ca/eng/document.jsp?did=97445.

${ }^{4}$ European Commission (2014) « Canada », modifié le 9 octobre 2014, http://ec.europa.eu/trade/policy/ countries-and-regions/countries/canada/.
} 
ont également débuté à la fin des années $1990^{5}$. Entre 2002 et 2006, le Canada a négocié sans succès un accord formel avec l'UE pour augmenter les relations bilatérales en matière de commerce et d'investissement. Il a fallu attendre la publication d'une étude des retombées potentielles, menée conjointement par le Canada et l'UE en 2008, avant que les négociations pour un Accord économique et commercial global entre les deux blocs ne soient lancées en 2009 (DEBLOCK ET RiOUX 2011: 52). Après de nombreux délais, les négociations pour l'AECG ont officiellement pris fin le 26 septembre 2014. Après une révision juridique finale, les deux parties auront à approuver, selon leurs propres procédures nationales, le texte final de l'Accord afin que celui-ci soit ratifié. Le délai dans lequel cela doit être effectué n'est toutefois pas spécifié dans le texte de l'Accord.

Les principaux objectifs de l'AECG pour Ottawa sont: l'ouverture de nouveaux marchés pour les entreprises canadiennes, la création d'emplois et la croissance économique $^{6}$. La littérature identifie aussi d'autres raisons pour lesquelles le Canada souhaite conclure l'AECG. Parmi celles-ci, nous retrouvons la volonté de réduire la dépendance vis-à-vis de l'ALÉNA qui, selon certains, a démontré des signes de faiblesses au fil du temps. Une autre raison donnée est la nécessité pour le Canada de se positionner et de participer à la prolifération des accords commerciaux multilatéraux, élaborés à l'extérieur du cadre de l'Organisation mondiale du commerce (OMC) (DEBLOCK ET RIOUX 2011: 40).

La Commission européenne partage avec le Canada les objectifs d'ouverture de nouveaux marchés, de création d'emplois et de croissance économique, liés à la consolidation des relations économiques et commerciales entre les deux parties. La volonté d'établir des partenariats commerciaux avec l'étranger, à l'extérieur du cadre de l'OMC, est également d'actualité pour l'UE, comme en témoignent notamment les négociations en cours avec les États-Unis pour le Partenariat transatlantique de commerce et d'investissement (PTCI). La littérature identifie trois autres facteurs motivant l'UE à négocier l'AECG. Tout d'abord, certains considèrent que la conclusion d'un accord économique avec le Canada permettra aux entreprises européennes qui s'installeront au Canada de

\footnotetext{
${ }^{5}$ L'Association européenne de libre-échange regroupe l'Islande, le Liechtenstein, la Norvège et la Suisse. Les négociations ont duré un peu plus d'une décennie. Un accord final est entré en vigueur seulement en juillet 2009 (DEBLOCK ET RIOUX 2011: 39, EFTA 2015).

${ }^{6}$ Affaires Etrangères Commerce et Développement Canada (2014a), «Canada-Union européenne : Accord économique et commercial global (AECG)», modifié le 4 novembre 2014. http://international.gc.ca/trade-agreements-accords-commerciaux/agr-acc/ceta-aecg/understandingcomprendre/overview-apercu.aspx?lang=fra.
} 


\section{Nassiba IDEBDOU et Sébastien LABRECQUE}

bénéficier d'un "effet plateforme ». Concrètement, un " effet plateforme » signifie qu'une entreprise européenne pourrait profiter de l'ALÉNA une fois installée au Canada. Cela lui donnerait ainsi un libre accès aux marchés des États-Unis et du Mexique, et ce, même si l'UE n'a pas d'accord de libreéchange avec ces deux pays à l'heure actuelle (BOULANGER ET MESSERLIN 2014: 277). Dans les faits, les bénéfices liés à un tel effet plateforme seront pertinents, tant et aussi longtemps que le PTCI ne sera pas en vigueur. Le partenariat ouvrira par la suite la porte des marchés américains et mexicains aux investisseurs européens sans qu'il soit nécessaire de passer par le Canada d'abord.

Les deux derniers facteurs sont directement en lien avec le sujet de cet article. Certains considèrent que l'AECG et le PTCI avec les États-Unis s'inscrit dans une logique délibérée de l'UE d'internationaliser les pratiques et les standards européens, afin de s'imposer sur la scène internationale en tant que chef de file en matière de règlementation globale («leading global regulatory actor») (HÜBNER 2011: 8). En d'autres mots, l'UE souhaiterait que les règlementations financières, proposées au niveau international, soient fortement similaires ou, au minimum, inspirées de celles déjà en place en Europe. Cela touche évidemment à la question de la convergence règlementaire dont nous discuterons davantage plus loin dans cet article.

Enfin, il est impératif de porter attention au désir des firmes européennes de pénétrer davantage le secteur des services au Canada. Parmi les domaines visés, la Commission Européenne identifie ouvertement: les télécommunications, l'énergie, le transport maritime et les services financiers ${ }^{7}$. Plus spécifiquement, au niveau des services financiers, les marchés des valeurs mobilières, des assurances et des banques attirent particulièrement l'intérêt des investisseurs et des firmes européennes (WoOLCOCK 2011:34). Ces sous-secteurs font tous l'objet d'articles du chapitre 15 de l'AECG sur les services financiers.

\section{2 - Portrait des secteurs financiers canadien et européen}

Le terme «secteur financier» regroupe plusieurs types d'activités: valeurs mobilières et investissement, assurances, crédit, services bancaires, etc. Nous nous concentrons sur le secteur bancaire dans cette sous-section, afin de dresser un bref portrait des secteurs financiers canadien et européen.

\footnotetext{
${ }^{7}$ European Commission (2014), op. cit.
} 
Au Canada, ce secteur est très fortement dominé par six banques (surnommées le Big 6) : Banque de Montréal, Banque Nationale, Banque Royale du Canada, Banque Scotia, Banque Canadienne Impériale de Commerce et Banque Toronto-Dominion. À cette liste, il faut ajouter, dans le cas du Québec, la Caisse Desjardins, bien qu'elle ne soit pas une banque à charte. En plus des activités bancaires traditionnelles, ces banques sont aussi autorisées, en vertu des lois fédérales, à opérer dans les domaines des services fonciers et fiduciaires, des assurances et des investissements (LÉTOURNEAU ET HEIDRICH 2010: 6). Cela contribue évidemment à la concentration du secteur financier au Canada. Il est à noter que toutes les activités des banques du Big 6 sont encadrées par le gouvernement fédéral du Canada. Cela permet au gouvernement de mieux contrôler le risque systémique dans le système bancaire canadien (BORDO, REDISH ET ROCKOFF 2011).

L'UE comprend 28 membres, il est donc quasi-impossible, dans le cadre de cet article, de décrire avec précision l'état du secteur bancaire dans chacun des pays. Cependant, au niveau européen, soulignons que quatre des dix plus grandes banques européennes en termes de capitalisation boursière, était d'origine britannique en date de juin 2014 : HSBC, Lloyds Banking Group, Royal Bank of Scotland et Barclays ${ }^{8}$. Pour ce qui est des activités auxquelles peuvent se livrer les banques européennes, cela varie à l'heure actuelle d'un pays à l'autre, selon les règlementations financières en place. Néanmoins, soulignons que l'on observe présentement une volonté au niveau de l'UE d'obliger les grandes banques à séparer leurs activités d'investissement de leurs services bancaires commerciaux traditionnels.

En plus des institutions financières nationales, on retrouve au Canada et en Europe un certain nombre de banques étrangères. La prochaine sous-section présente l'ampleur plutôt limitée de la pénétration d'institutions non-nationales dans ces pays, comparativement aux États-Unis et ses banques.

\section{2-1 : Pénétration des institutions financières européennes au Canada (et vice versa)}

La concentration du secteur bancaire au Canada fait en sorte qu'il est difficile pour les banques étrangères de pénétrer le marché et de rivaliser avec les établissements canadiens. Néanmoins, on remarque que les firmes de services

\footnotetext{
${ }^{8}$ SNL Financial (2014), "SNL Financial ranks the top 20 European banks by market capitalization », SNL Financial, http://ofccolo.snl.com/cache/24786002.pdf.
} 


\section{Nassiba IDEBDOU et Sébastien LABRECQUE}

financiers américaines ont mieux réussi à s'établir au Canada que leurs homologues européens. À la fin de 2013, on comptait seulement 11 institutions financières européennes enregistrées au Canada contre 21 entités américaines sur un total de 73 institutions au Canada incluant les firmes canadiennes ${ }^{9}$.

Pour ce qui est du taux de pénétration des institutions financières canadiennes à l'étranger, on remarque que le marché des États-Unis est de loin beaucoup plus développé que celui de l'Europe. En effet, on dénombre un total de 39 institutions canadiennes enregistrées aux Etats-Unis, alors que ce nombre chute de plus de la moitié pour le marché européen pour s'établir à 15 entités canadiennes enregistrées ${ }^{10}$.

Ces données illustrent bien le fait que l'objectif des banques canadiennes se trouve davantage sur le marché des États-Unis que sur celui de l'Europe. Il semble également que les institutions financières européennes n'ont pas réussi à bien s'installer au Canada jusqu'à maintenant. Cela pourrait éventuellement changer puisque, comme nous l'avons mentionné, les Européens considèrent l'AECG comme une occasion d'augmenter leur taux de pénétration du marché canadien des services financiers. Ce désir pourrait se heurter à la domination du Big 6 ainsi qu'au fait que le marché bancaire canadien soit déjà saturé selon certains auteurs (LÉTOURNEAU ET HEIDRICH 2010: 2).

\section{2-2 : Règlementation des secteurs financiers au Canada et de l'Union européenne}

\section{Qui règlemente les secteurs financiers : conséquences pour l'AECG}

La règlementation des services financiers au Canada est généralement considérée comme relativement centralisée pour les activités bancaires et excessivement décentralisée pour les assurances et les valeurs mobilières. Cela s'explique par le fait que les banques à charte sont régies par les lois fédérales en vertu de la constitution canadienne. Les principaux organismes de règlementation sont le Ministère des finances, la Banque du Canada et le Bureau du surintendant des institutions financières. Ces derniers collaborent constamment entre eux.

\footnotetext{
${ }^{9}$ Bank for International Settlements (2014), «Locational banking statistics : List of reporting institutions at end-December 2013 By country of location ", page consultée le 10 avril 2015, www.bis.org/statistics/locstatsrepinst.xls.

${ }^{10}$ Ibid.
} 
La régulation des assurances et des valeurs mobilières est pour sa part une compétence provinciale. Cela signifie que chaque province a sa propre agence de règlementation dans ce domaine. Comme le soulignait Stephen Woolcock en 2011, cette fragmentation règlementaire au niveau des valeurs mobilières pose problème à des firmes de service financier européennes puisqu'elles doivent obtenir l'approbation de chacune de ces agences pour opérer au Canada contrairement à l'UE où l'obtention d'une approbation dans un pays membre donne généralement accès aux marchés des autres États (WoOLCOCK 2011 : $34)$.

Il est à noter que l'AECG ne remédie point à cette fragmentation et ne prévoit pas la mise en place d'un "passeport commun» comme les négociateurs européens l'auraient souhaité. Au contraire, l'article X-03 du chapitre 11 sur le commerce transfrontalier des services autorise les parties à exiger des établissements l'obtention d'enregistrement et/ou de permis pour fournir certains services financiers. Au Canada, dans les cas des assurances et des valeurs mobilières, ces enregistrements et/ou permis sont accordés par des organismes règlementaires provinciaux comme la Commission des valeurs mobilières de l'Ontario et l'Autorité des marchés financiers au Québec. Cela signifie que le Canada pourra techniquement conserver son architecture règlementaire dans ces domaines. Il reste maintenant à savoir si les organismes règlementaires provinciaux décideront éventuellement de collaborer entre eux, afin de faciliter la reconnaissance des permis entre les provinces. Réduire la fragmentation règlementaire faciliterait évidemment les choses pour les firmes étrangères souhaitant s'installer au Canada en leur permettant, entre autre, d'opérer sur l'ensemble du marché canadien.

Au niveau de l'UE, les États membres conservent traditionnellement un rôle dans la règlementation des services financiers. Le système d'approbation et de reconnaissance entre pays est toutefois relativement mieux développé qu'au Canada: obtenir l'approbation pour un service financier dans un pays donne généralement accès aux marchés de plusieurs autres pays de l'UE. Il existe toutefois certaines limites à cette reconnaissance dans le secteur bancaire. En effet, le Canada a tenté d'obtenir, lors des négociations de l'AECG, que la mise en place d'une succursale dans un pays de l'UE permette à une banque canadienne d'utiliser ce «pied à terre " pour s'installer librement dans d'autres pays de l'UE par la suite, grâce au marché commun européen. Cette demande du Canada n'a toutefois pas été intégrée à l'AECG puisqu'elle était en contradiction avec les lois européennes qui considèrent que les firmes non incorporées comme les succursales de banques ne constituent pas un «pied à 


\section{Nassiba IDEBDOU et Sébastien LABRECQUE}

terre » valide, permettant l'expansion à travers l'Europe. Il est en ce sens impossible pour le Canada de bénéficier d'un «effet de plateforme » dans ce secteur.

$\mathrm{Au}$ niveau national se superpose le niveau de règlementation financière européen, qui lui, s'est plus particulièrement développé suite à la crise économique de 2008. Par exemple, une partie de la règlementation des banques a été centralisée au niveau supranational, avec la mise en place d'une union bancaire jumelée à des mécanismes de surveillance et de résolution unique. En ce sens, on peut affirmer que l'UE s'est rapprochée du modèle canadien après 2008, en tentant de centraliser au niveau fédéral l'encadrement des banques. Ces développements règlementaires européens ont toutefois eu comme conséquence de compliquer la tâche des négociateurs canadiens puisque cette transition institutionnelle s'opérait en Europe au même moment où Bruxelles tentait de développer l'AECG. Au niveau de l'UE, certaines dispositions comme, par exemple, les mécanismes de dispute investisseurs-états et de reconnaissance mutuelle étaient relativement mal définis et encore en processus de finalisation au moment des négociations. Cela explique pourquoi l'UE n'a pas réellement cherché à faire de celles-ci des éléments clés et importants de 1'AECG et, plus précisément, du chapitre $15^{11}$. Cette période de changements en matière de règlementation financière que vivait l'UE lors des négociations avec le Canada a contribué à ce que le chapitre 15 de l'AECG soit somme toute assez « classique » en étant très proche des dispositions de l'Accord général sur le commerce des services.

\section{Le Canada est-il plus sévère en matière de règlementations financières?}

Les règlementations canadiennes en matière de services financiers sont généralement décrites comme étant plus sévères que celles appliquées en Europe. Dans le domaine bancaire, le Canada exigeait, avant la crise de 2008, des niveaux de fonds propres règlementaires plus élevés que ceux suggérés par l'Accord de Bâle II (LEBLOND 2010: 69). Suite à 2008, il est à noter que le Canada a adopté les règles du nouvel Accord de Bâle III $^{12}$ plus rapidement que les délais autorisés par le texte de l'entente.

\footnotetext{
${ }^{11}$ Il est à noter que, dans le cadre des négociations du PTCI, l’UE tente de faire accepter aux ÉtatsUnis un texte plus ambitieux que l'AECG en matière de mécanismes de dispute et de reconnaissance mutuelle. Les Européens font toutefois face à une résistance marquée des Américains sur cet enjeu.

${ }^{12}$ Certaines des mesures prévues par Bâle III s'inscrivent directement dans la logique des règlementations canadiennes qui étaient déjà en place avant la crise (comme les niveaux de réserve
} 
La crise de 2008 et l'élan règlementaire qui a eu lieu au niveau global ont encouragé l'UE à réviser et à renforcer ses règlementations financières, notamment en matière de capitalisation bancaire obligatoire, puisque les banques européennes étaient hautement sous-capitalisées avant la crise de 2008. On observe que l'UE adopte généralement de manière assez fidèle les lois qui sont développées dans les forums financiers internationaux comme le Conseil de stabilité financière (CSF) et la Banque des règlements internationaux (BRI). Par exemple, l'Europe est sensiblement à la même étape de mise en œuvre que le Canada en ce qui concerne les dispositions de l'Accord de Bâle III et a été très rapide à appliquer les recommandations du CSF liées au système bancaire parallèle. Contrairement au Canada, l'UE ne tend toutefois pas à aller au-delà des mesures proposées au niveau global. Cela est notamment attribuable au Royaume-Uni qui accepte généralement de collaborer avec Bruxelles en matière de règlementations financières, tant que celles-ci ne sont pas plus sévères que celles développées dans les forums internationaux. Des mesures n'émanant pas de ces forums comme, par exemple, le règlement délégué de la Commission européenne sur les gestionnaires de fonds d'investissement alternatifs en 2009 et le projet de taxe sur les transactions financières, s'attirent généralement une farouche opposition de la part du Royaume-Uni.

En résumé, il est effectivement possible d'affirmer que le Canada était plus sévère en matière de règlementations financières que les pays européens, avant la crise économique de 2008. Les choses ont cependant beaucoup évolué depuis ce temps et l'UE a fait un impressionnant rattrapage dans ce domaine pour adopter les politiques et les exigences proposées au niveau international.

Ce portrait des secteurs financiers du Canada et de l'UE que nous venons de présenter constitue la toile de fonds sur laquelle le chapitre 15 de l'AECG sera appliqué. S'il est vrai que le portrait actuel des secteurs financiers du Canada et de l'UE a façonné l'élaboration du chapitre 15, il est également possible d'avancer que ce chapitre influencera à son tour les secteurs financiers de ces pays.

plus élevés et les ratios de levier). Cela contribue notamment à expliquer pourquoi le Canada a pu s'ajuster aux exigences de Bâle III relativement rapidement. 


\section{Nassiba IDEBDOU et Sébastien LABRECQUE}

\section{3 - Chapitre 15 de l'AECG sur les services financiers}

Cette section analyse les impacts potentiels du chapitre 15 de l'AECG en ayant recours au concept de convergence règlementaire. Cette section étudie également des articles de l'Accord portant sur la possibilité d'appliquer des exceptions prudentielles et sur la mise en place d'un Comité des services financiers regroupant des représentants du Canada et de l'UE

Le chapitre 15 de l'AECG contient une vingtaine d'articles et trois annexes. Pour les fins de l'AECG, le terme service financier est défini de la façon suivante :

«Service financier » désigne tout service de nature financière. Les services financiers incluent tous les services d'assurance et services connexes, et tous les services bancaires et autres services financiers (l'assurance exclue), ainsi que les services auxiliaires ou accessoires à un service de nature financière ${ }^{13}$.

Cette définition met en relief que les secteurs des assurances et des banques constituent les deux grands axes du chapitre $15^{14}$. Il s'agit des deux principaux types de services financiers pour lesquels les investisseurs européens ont des visées au Canada. Cependant, il ne faut pas conclure pour autant que ces deux secteurs ont été intégrés à l'AECG simplement pour satisfaire les Européens. Dans les faits, les assurances et les banques constituent également les deux principaux champs d'activités de la définition de « services financiers » donnée par l'Annexe sur les services financiers de l'AGCS signée sous les auspices l'OMC et entrée en vigueur au milieu des années 1990. Il est à noter que les définitions de "services d'assurance » et de "services bancaires 》 utilisées dans le cadre de l'AECG entre le Canada et l'UE (article 2(a) A et B) sont essentiellement les mêmes que celles qui se retrouvent dans l'Annexe sur les services financiers de l'AGCS (article 5(a)). L'influence de l'AGCS sur le chapitre 15 de l'AECG a d'ailleurs été soulignée par Patrick Leblond de l'Université d'Ottawa en octobre 2014. Celui-ci avançait que les dispositions du chapitre 15 sur les services financiers de l'Accord Canado-européen s'inscrivaient, pour la majorité, dans la lignée de celles que l'on retrouve dans

\footnotetext{
${ }^{13}$ Les valeurs mobilières font partie de la définition des services bancaires en vertu de l'accord. AECG, chapitre 15, article 2(a).

${ }^{14} \mathrm{Il}$ est à noter, qu'en vertu de l'article 16 du chapitre 15 , les mesures liées à la politique monétaire et au taux de change ne sont pas couverts par l'accord. Les États conservent donc leur souveraineté dans ce domaine.
} 
l'AGCS et l'ALÉNA (LEBLOND 2014). Cet argument nous amène à nous interroger sur les retombées qu'aura le chapitre 15 de l'AECG puisque, comme nous l'avons mentionné plus tôt, le niveau de pénétration au Canada des firmes de services financiers européennes (et vice versa en Europe pour les firmes canadiennes) est significativement moins élevé que celui des compagnies américaines, et ce, malgré le fait que l'AGCS régit, depuis le milieu des années 1990, les investissements dans les services financiers entre le Canada et l'Europe.

Le Canada et les pays de l'UE sont tous signataires de l'AGCS en leur qualité de membres de l'OMC. En ce sens, l'adaptation aux mesures prévues par le chapitre 15 de l'AECG devrait donc en théorie être facilitée puisque plusieurs dispositions sont censées déjà être intégrées en droit national, en vertu de l'AGCS. Cela soulève évidemment un enjeu important, à savoir si le fait d'être membre des mêmes cadres règlementaires internationaux et des mêmes organisations et forums internationaux, mène en pratique à une convergence règlementaire. Des auteurs comme Daniel Drezner, Xun Cao, Martha Finnemore et Kathryn Sikkink avancent que les organisations internationales contribuent d'une manière ou d'une autre à une telle convergence. Finnemore et Sikkink perçoivent les organisations internationales comme des agents de socialisation qui poussent les États à adopter les politiques qu'elles élaborent. La nature de ces politiques publiques varie selon le champ d'expertise de l'organisation internationale. Ces organisations peuvent également surveiller le respect de standards internationaux (FINNEMORE ET SIKKINK 1998: 902). Xun Cao accepte le rôle « social» des organisations internationales et avance que « the more common IGO [intergovernmental organization] memberships that two countries share, the smaller the policy distance between them, which implies more similar profiles of domestic economic policies » (CAO 2012: 407).

Cela s'expliquerait notamment par les processus d'apprentissage et d'émulation que rendent possibles les interactions entre les États dans le cadre d'instances internationales (CAO 2012: 407). Enfin, se penchant sur les standards environnementaux et du travail, Daniel Drezner accorde plus d'agence à l'État en soulignant que l'harmonisation des politiques publiques dans différents domaines et donc la convergence règlementaire demeure un choix que font consciemment les gouvernements, sous les auspices des organisations internationales où les grands cadres règlementaires globaux et recommandations sont élaborés (DREZNER 2001: 55). 


\section{Nassiba IDEBDOU et Sébastien LABRECQUE}

Les conclusions de ces auteurs peuvent évidemment être transposées au cas du Canada et de l'UE. Suivant l'argument de Xun Cao, les deux parties sont effectivement membres des mêmes forums règlementaires internationaux dans le secteur de l'économie et de la finance. Pensons notamment à l'Organisation mondiale du commerce, au Fonds monétaire international, à l'Organisation internationale des commissions des valeurs, au CSF et à la BRI. L'idée de Cao ne peut cependant pas expliquer à elle seule les différences dans l'ensemble des règlementations financières que les gouvernements appliquent au niveau national. Garder au cour de l'analyse la notion d'agence de l'État, comme le fait Drezner, semble crucial. Certes, le Canada et l'UE tiennent compte des recommandations et des cadres règlementaires que développent les organisations internationales, mais ces derniers constituent avant tout des lignes directrices à partir desquelles les gouvernements canadien et européens établissent leurs propres politiques en étant parfois plus sévère. En pratique, les États demeurent libres d'altérer ou de ne pas appliquer certaines dispositions prévues dans des accords internationaux. Cela est particulièrement vrai lorsque ces mesures émanent d'organisations n'ayant pas de réel pouvoir de cohésion, comme notamment le CSF.

Dans le cas précis du chapitre 15 de l'AECG, un double argument peut être évoqué. Premièrement, dans la mesure où le chapitre est relativement similaire aux dispositions de l'AGCS, on pourrait affirmer que l'adhésion du Canada et celle de l'UE à l'OMC ont déjà contribué à un processus de convergence règlementaire entre les deux blocs. La prochaine sous-section analyse les bénéfices et les coûts associés à la convergence règlementaire et ce que cela signifie dans le contexte de l'AECG.

Deuxièmement, on remarque aussi que les gouvernements ont été soucieux de conserver une certaine agence en intégrant à l'AECG la possibilité d'appliquer au niveau national des exceptions prudentielles en matière de règlementation des services financiers. Ces mesures prudentielles sont étudiées à la section 3.2.

\section{3-1 : Sur les bénéfices et les coûts de la convergence règlementaire}

La littérature identifie un certain nombre de coûts et de bénéfices associés à la convergence règlementaire. Il est généralement considéré que la coordination règlementaire diminue les coûts de transactions pour les firmes en leur permettant notamment d'être soumis à des règlementations et des exigences administratives relativement similaires d'un pays à l'autre (DREZNER 2005). Ces facteurs peuvent faciliter la tâche à des investisseurs souhaitant s'installer 
sur des marchés étrangers: ceux-ci peuvent ainsi avoir des processus de production uniformisés puisqu'ils n'ont pas à composer avec des exigences différentes d'un pays à l'autre.

La coordination règlementaire permet également de préciser les processus par lesquels les standards règlementaires peuvent être modifiés (DREZNER 2005: 845). C'est d'ailleurs l'un des rôles attribués au Comité des services financiers créé par le chapitre 15 de l'AECG. En effet, en vertu de la section «Examen périodique » de l'Annexe XX, le Comité dispose du pouvoir de modifier le contenu du chapitre 15 avec l'accord du Canada et de l'UE. Nous présentons ce Comité des services financiers plus en détails à la sous-section 3.3.

La convergence règlementaire implique également des coûts pour les États et les acteurs privés. Selon Daniel Drezner, la coordination règlementaire et l'adoption de nouvelles politiques qui sont souvent nécessaires à cause de cette convergence, impliquent des coûts économiques et politiques pour les gouvernements. Celui-ci identifiait dans un article publié en 2005 les coûts suivants:

Governments incur costs from the retraining of regulators, and from the restructuring or creation of new regulatory infrastructures. They also incur the political costs of getting new standards ratified by other branches of the state, or from dissatisfaction with the new standards among voters, interest groups, or members of the electorate. (DREZNER 2005: 845).

Considérant ces coûts pour les pouvoirs publics, Drezner avance l'argument que l'option préférée des gouvernements est que leurs standards règlementaires nationaux soient ceux utilisés dans le cadre d'accords et/ou de forums multilatéraux, pour limiter leurs coûts d'adaptation (DREZNER 2005: 846). Enfin, soulignons que les entreprises privées encourent également des coûts lorsqu'il y a convergence règlementaire puisqu'elles doivent elles aussi modifier leurs façons de faire pour s'adapter aux nouvelles exigences règlementaires. Pour limiter ces coûts d'adaptation, les firmes préfèrent, à l'instar des gouvernements, que les normes nationales s'imposent au niveau international (DREZNER 2005: 845).

Ces bénéfices et ces coûts liés à la convergence sont pertinents lorsque l'on étudie les impacts de l'AECG. Dans le cas du chapitre 15 de l'Accord canadoeuropéen, certains des bénéfices identifiés peuvent être transposés, dans une 


\section{Nassiba IDEBDOU et Sébastien LABRECQUE}

certaine mesure, au secteur des services bancaires ainsi qu'aux domaines des assurances et des valeurs mobilières en Europe. Cependant, la fragmentation règlementaire au Canada dans les secteurs de l'assurance et des valeurs mobilières, signifie que des coûts de transaction persisteront, du moins à courtmoyen terme, pour les investisseurs européens souhaitant faire des affaires sur les marchés canadiens. Il est à noter que la mise en place d'exceptions prudentielles autant par le Canada que par l'UE pourrait également imposer des coûts de transactions aux firmes de services financiers. Pour ce qui est des coûts liés à la convergence identifiés par Drezner, ils ne devraient pas être un facteur majeur pour les gouvernements et les firmes dans le cas du chapitre 15, puisque la majorité des dispositions de celui-ci sont inspirées très fortement de l'AGCS qui est déjà en place depuis 20 ans.

\section{3-2 : Exceptions prudentielles}

Le fait que les services financiers soient intégrés à l'AECG ne signifie pas que les gouvernements nationaux perdront toute possibilité de règlementer les services financiers au niveau national. L'article 15 du chapitre 15 autorise les États à adopter des mesures prudentielles qui ne sont pas stipulées par l'AECG $^{15}$. Le texte de l'article identifie les raisons suivantes pour justifier l'application d'exceptions prudentielles :

Aucune disposition du présent accord n'empêche une Partie d'adopter ou de maintenir des mesures raisonnables pour des raisons prudentielles, notamment :

a) la protection des investisseurs, des déposants, des titulaires de police ou des personnes à l'égard desquelles une institution financière ou un fournisseur de services financiers transfrontières a des obligations fiduciaires;

b) le maintien de la sécurité, de la solidité, de l'intégrité ou de la responsabilité financière des institutions financières, des fournisseurs de services financiers transfrontières ou des fournisseurs de services financiers;

c) la préservation de l'intégrité et de la stabilité du système financier d'une Partie (AECG, article 15.1).

L'AECG prévoit aussi des recours permettant à un investisseur de porter plainte pour critiquer une exception prudentielle d'un État si celui-ci estime que la

${ }^{15}$ Cela vient s'ajouter aux exceptions qui sont déjà spécifiées explicitement dans le texte du chapitre 15. 
mesure va au-delà de ce qui est nécessaire ou justifiable pour "préserver la stabilité du système financier $»^{16}$. Ces recours impliqueront d'abord le Comité des services financiers que nous analyserons dans la prochaine section ou le Comité sur les échanges commerciaux, et, en deuxième instance, un tribunal qui sera constitué pour gérer un différend précis ${ }^{17}$.

Les raisons justifiant le recours à des exceptions prudentielles sont relativement larges et les termes choisis dans l'article sont vagues. En effet, plusieurs mesures peuvent potentiellement être justifiées par l'expression "préservation de l'intégrité et la stabilité du système financier ». Qui plus est, l'utilisation du mot «notamment» à l'article 15.1 indique qu'il ne s'agit pas d'une liste exhaustive et que les gouvernements pourraient potentiellement utiliser d'autres raisons pour justifier le recours à des exceptions prudentielles.

Il est à noter que les exceptions prudentielles sont également permises par l'AGCS. Les raisons mentionnées à l'article 15.1(a) et (c) du chapitre 15 de l'AECG sont essentiellement identiques aux raisons identifiées par l'AGCS tandis que l'article 15.1(b) se trouve uniquement dans l'AECG. En ce sens, il est intéressant de souligner qu'une portion de la littérature sur l'AGCS fait état de l'imprécision de la notion de mesure prudentielle destinée à préserver la stabilité du système financier (YOKOI-ARAi 2008, MeEsTER 2010). Le principal enjeu lié à la vague utilisation du terme « exception prudentielle» dans le cas de l'AGCS est qu'il a rendu la surveillance et le contrôle du respect des dispositions de l'accord relativement difficile (YOKOI-ARAI 2008: 640). À la lumière des similitudes entre l'AGCS et l'AECG en matière d'exception prudentielle, l'argument de Yokoi-Arai peut potentiellement être transposé au cas du chapitre 15 de l'AECG entre le Canada et l'UE qui lui aussi offre une notion de "mesure prudentielle» imprécise et peu définie. Qui plus est, l'article 15 du chapitre 15 de l'AECG inclut plus de façons de justifier des mesures prudentielles que l'AGCS.

\footnotetext{
${ }^{16}$ Pour les lignes directrices identifiées par l'AECG pour établir si une exception prudentielle est légitime ou non, voir la sous-section «Principes de haut niveau » de l'Annexe XX du chapitre 15.

${ }^{17}$ Voir l'article 20 et la sous-section «procédure » de l'Annexe XX du chapitre 15 pour davantage de détails concernant les procédures de règlement des différends en matière d'investissement pour les services financiers.
} 


\section{Nassiba IDEBDOU et Sébastien LABRECQUE}

\section{Exceptions prudentielles : conséquence sur la sévérité des règlementations financières}

Nous avons souligné plus tôt dans cet article que le Canada a historiquement eu tendance à appliquer des règlementations financières plus sévères que celles proposées au niveau international. Le Canada devrait en théorie être en mesure de continuer dans cette direction dans le cadre de l'AECG avec l'Europe. La notion de préservation de la stabilité du système financier pourrait certes être invoquée par Ottawa pour justifier certaines mesures prudentielles. Cependant, une disposition de l'AECG dans l'Annexe XX (article 1 de la sous-section «Principes de haut niveau ») souligne plus clairement la possibilité d'appliquer des règlementations plus sévères :

Chaque Partie peut définir son propre niveau approprié de règlementation prudentielle. Plus particulièrement, une Partie peut établir et appliquer des mesures qui lui confèrent un niveau de protection prudentielle supérieur à ceux qui sont établis dans les engagements prudentiels internationaux communs.

En protégeant les gouvernements qui souhaiteraient appliquer des mesures prudentielles plus sévères en matière de services financiers, le chapitre 15 réduit la possibilité qu'un nivèlement par le bas $^{18}$ (« race to the bottom ») se produise. Si les mesures prudentielles plus strictes n'étaient pas tolérées dans le cadre de l'AECG, cela créerait un incitatif pour les États d'adopter des règlementations financières, les moins sévères possible. Deux raisons expliquent cet incitatif. Premièrement, sans la possibilité d'invoquer la clause sur les mesures prudentielles pour se justifier, les règlementations financières d'un État participant à l'AECG seraient toujours à risque de faire l'objet de plaintes pour traitement injuste et/ou obstacle au libre commerce de la part des investisseurs. Deuxièmement, toujours suivant une logique de nivèlement par le bas, adopter des règlementations moins sévères que les autres participants à l'AECG pourrait permettre à un pays donné d'attirer davantage d'entreprises et de capitaux étrangers que ses homologues aux critères plus strictes.

À l'inverse, les exceptions prudentielles pourraient potentiellement aussi permettre à un gouvernement donné de compliquer l'accès à son marché

\footnotetext{
${ }^{18}$ Un nivèlement par le bas décrit une situation où les pays adoptent des réglementations les moins sévères possible afin d'inciter les entreprises à s'installer sur leurs territoires. Le postulat de base du concept de nivèlement par le bas est que les entreprises et investisseurs cherchent toujours à s'installer là où ils sont soumis au moins d'encadrement possible.
} 
national, aux banques étrangères en ayant des exigences règlementaires plus sévères que les standards internationaux. Les investisseurs étrangers auraient donc des coûts d'ajustement (une sorte de coût à l'entrée ou " protectionnisme règlementaire ») dont les compagnies déjà installées sur le territoire n'auraient pas à se préoccuper. Il sera donc intéressant de voir comment cette disposition de l'AECG sera interprétée par le Comité des services financiers, le Comité sur les échanges commerciaux et peut-être les tribunaux dans les cas où des investisseurs décideront de tester la portée de cet article en portant formellement plainte.

\section{Exceptions prudentielles : conséquence sur la convergence règlementaire}

Enfin, soulignons que les exceptions prudentielles devraient permettre aux gouvernements canadien et européens de tempérer, jusqu'à un certain point, la convergence règlementaire que l'AECG pourrait générer en protégeant certains éléments de leurs systèmes financiers respectifs. Il est vrai que les procédures de règlement des différends de l'AECG pourraient possiblement s'avérer une force poussant à la convergence règlementaire en matière de services financiers, notamment en ne reconnaissant pas la légitimité des exceptions prudentielles adoptées au niveau national. Cependant, il faut garder en tête que ces mécanismes ne s'attaqueront aux exceptions prudentielles que dans les cas où des plaintes seront déposées par des investisseurs. En d'autres mots, une mesure prudentielle sera légale jusqu'à preuve du contraire.

Il est en ce sens intéressant de constater que des acteurs privés comme les investisseurs constitueront l'un des catalyseurs du processus de convergence règlementaire, par le fait qu'ils détiennent le pouvoir de déclencher les procédures de règlement des différends prévu par l'AECG.

\section{3-3 : Le Comité des services financiers}

L'article 17 du chapitre 15 de l'AECG instaure le Comité des services financiers. Conformément à l'article 17.2, cette entité regroupera des représentants des diverses autorités responsables de la règlementation des services financiers au Canada et de l'UE. Il est à noter que le texte consolidé de l'Accord ne précise toujours pas le nombre exact de représentants qui siègeront sur le Comité. En plus d'être en charge de superviser l'application des dispositions du chapitre 15 de l'AECG, le Comité des services financiers sera, comme nous l'avons mentionné précédemment, directement impliqué dans le mécanisme de règlement des différends dans les cas où les plaintes porteront 


\section{Nassiba IDEBDOU et Sébastien LABRECQUE}

sur les exceptions prudentielles. Le Comité étant en première ligne du processus de plainte, celui-ci aura un rôle crucial de triage. Conformément à l'article 20.4, une plainte sera considérée comme ayant été retirée par l'investisseur si le Comité décide en première instance que les mesures contestées sont effectivement des exceptions prudentielles légitimes. Un tribunal sera constitué uniquement dans les cas où le Comité estime que l'article 15 ne constitue pas une justification totalement appropriée pour une règlementation financière donnée.

L'une des principales difficultés à laquelle sera for probablement confronté le Comité des services financiers, sera de parvenir à prendre des décisions par consensus, comme l'exige l'article $17.3 \mathrm{du}$ chapitre 15. Deux facteurs pourraient compliquer l'obtention de décision par consensus et potentiellement ralentir, voire même paralyser le Comité ${ }^{19}$. Premièrement, les représentants siégeant au Comité seront-ils capables de se dissocier de leur citoyenneté, pour être totalement neutres et objectifs lorsqu'ils auront à se prononcer dans les dossiers où un investisseur étranger portera plainte contre des mesures prudentielles adoptées par le gouvernement national qu'ils représentent ? Derrière cette question se trouve aussi l'enjeu de savoir si ces représentants et leurs positions feront l'objet de pressions politiques. Deuxièmement, obtenir un consensus pourrait s'avérer complexe dans un contexte où les régulateurs canadiens et européens n'ont pas nécessairement la même culture règlementaire en matière de services financiers. Selon certains, le Canada et l'UE ont généralement eu tendance à adopter des politiques règlementaires relativement différentes en matière d'assurance et de services bancaires (LEBLOND 2010: 71). Il n'existe pas de tradition de coopération directe entre les deux blocs dans le domaine de la règlementation financière. Avant l'avènement de l'AECG et du chapitre 15, le Canada et l'UE collaboraient généralement seulement dans le cadre d'organisations et de forums internationaux (LEBLOND 2010).

L'article 17.4 (b) met en relief l'objectif selon lequel le Comité des services financiers ouvre les lignes de communication entre Bruxelles et Ottawa :

4. Le Comité se réunit chaque année, ou à une autre périodicité convenue, et il s'occupe: [...] (b). de favoriser un dialogue sur la règlementation $\mathrm{du}$ secteur des services financiers, dans le but

\footnotetext{
${ }^{19}$ Il est à noter qu'en vertu de l'article 5 de la sous-section «Procédure » de l'Annexe XX, les différends qui ne font pas l'objet d'une décision par le Comité des services financiers au bout de 60 jours (notamment parce qu'un consensus n'a pas été atteint) seront transférés au Comité sur les échanges commerciaux de l'AECG.
} 
d'améliorer les connaissances mutuelles des systèmes de règlementation respectifs et de collaborer à l'élaboration de normes internationales, comme il est illustré dans la déclaration d'interprétation se trouvant à l'annexe X $[\ldots]$.

Le Comité, en tant qu'instance, devrait donc contribuer à moyen-long terme à développer une culture règlementaire commune entre les régulateurs canadiens et européens. Cela semble d'ailleurs être l'un des objectifs de l'AECG. Un tel processus prendra du temps. Dans l'immédiat, il semble peu probable que le Comité s'avère un acteur cohérent et influent. Sur le papier, le Comité possède certains pouvoirs lui permettant d'encourager une certaine convergence règlementaire entre les deux parties de l'AECG. En pratique, sa capacité à pousser vers une telle convergence sera tributaire de la coopération entre les représentants canadiens et européens siégeant à celui-ci et du développement d'une culture règlementaire partagée par ces acteurs. L'importance d'une culture commune ne doit pas être sous-estimée puisqu'elle se retrouve dans une certaine mesure dans la littérature sur la convergence, sous la forme de variables idéationnelles. La formation des idées et, plus important encore, le partage de celles-ci sont en effet identifiés comme l'un des moteurs du processus de convergence règlementaire (DREZNER 2001).

\section{Conclusion}

Cet article tentait d'analyser quels seraient les impacts potentiels du chapitre 15 de l'AECG sur les secteurs financiers canadien et européen et sur la règlementation de ceux-ci. Pour répondre à cette question, ont d'abord été brièvement présentées les grandes caractéristiques de l'AECG, puis un portrait des secteurs financiers du Canada et de l'UE et de la règlementation de ces derniers. La dernière section s'attardait précisément sur le chapitre 15. Les bénéfices et les coûts identifiés dans la littérature, sur la convergence règlementaire ont d'abord été présentés et transposés au cas précis de l'AECG. Les deux dernières sous-sections de l'article ont étudié les exceptions prudentielles permises par l'AECG et le Comité des services financiers.

En se basant sur la notion de convergence règlementaire ainsi qu'en considérant les similitudes entre le chapitre 15 et l'AGCS, et la possibilité d'adopter des exceptions prudentielles, nous confirmons la thèse selon laquelle l'AECG ne devrait pas mener à une convergence règlementaire majeure entre le Canada et l'UE en matière de services financiers, et donc, que l'impact du chapitre 15 sur les secteurs financiers des deux blocs sera limité, à court-moyen terme. Si une 


\section{Nassiba IDEBDOU et Sébastien LABRECQUE}

convergence règlementaire devait se matérialiser à moyen-long terme, il semble probable qu'elle proviendra du Comité des services financiers. Plus d'attention et d'analyse devront être portées, lors des années à venir, à la règlementation des assurances et des valeurs mobilières. Il sera en effet intéressant de voir comment les investisseurs européens se comporteront face aux règlementations canadiennes fragmentées en matière d'assurance et de valeurs mobilières. Nous verrons en ce sens si une certaine convergence au niveau interprovincial prendra éventuellement forme pour faciliter l'accès aux firmes étrangères de services financiers.

\section{Bibliographie}

Affaires Étrangères, Commerce et Développement Canada (2014a), « CanadaUnion européenne: Accord économique et commercial global (AECG) », modifié le 4 novembre 2014.http://international.gc.ca/trade-agreementsaccords-commerciaux/agr-acc/ceta-aecg/understanding-comprendre/overviewapercu.aspx?lang=fra.

Affaires Étrangères, Commerce et Développement Canada (2014b), « Texte de l'AECG consolidé - 15. Services financiers », modifié le 25 septembre 2014. http://www.international.gc.ca/trade-agreements-accords-commerciaux/agracc/ceta-aecg/text-texte/15.aspx?lang=fra.

Band for International Settlements (2014), « Locational banking statistics : List of reporting institutions at end-December 2013 By country of location », page consultée le 10 avril 2015, www.bis.org/statistics/locstatsrepinst.xls.

BORDO, Michael D., REDISH, Angela and ROCKOFF, Hugh (2011), "Why didn't Canada have a banking crisis in 2008 (or in 1930, or 1907, or ...)?», NBER Working Paper Series, National Bureau of Economic Research.

BOULANGER, Pierre et MESSERLIN, Patrick (2014), «La politique commerciale européenne: Entre recherche de stabilité politique et de croissance économique ", Revue de l'OFCE 3(134), pp. 269-279.

CAO, Xun (2012), «Global Networks and Domestic Policy Convergence: A Network Explanation of Policy Changes », World Politics 64(3), pp. 375-425. 
DEBLOCK, Christian and RIOUX, Michèle (2010), « From economic dialogue to CETA: Canada's trade relations with the European Union », International Journal 66(1), pp. 39-56.

Deutsche Bundesbank (2014), « The shadow banking system in the euro area: overview and monetary policy implications ", Deutsche Bundesbank Monthly Report, March 2014, https://www.bundesbank.de/Redaktion/EN/Downloads/Publications/ Monthly Report Articles/2014/2014 03 shadow.pdf? blob=publicationFile.

DREZNER, Daniel W. (2001), "Globalization and Policy Convergence », International Studies Review 3(1), pp. 53-78.

--- (2005), «Globalization, Harmonization, and Competition: the Different Pathways to Policy Convergence », Journal of European Public Policy 12(5), pp. 841-859.

DUCHESNE, Érick \& MORIN, Jean-Frédéric (2013), «Revisiting Structural Variables of Trade Negotiations: The Case of the Canada-EU Agreement », International Negotiation 18, pp. 5-24.

European Free Trade Association, EFTA (2015), «Free Trade Agreements Canada », page consultée le 8 avril 2015, http://www.efta.int/free-trade/freetrade-agreements/canada.

European Commission (2014) "Canada», modifié le 9 octobre 2014, http://ec.europa.eu/trade/policy/countries-and-regions/countries/canada/.

FINNEMORE, Martha and SIKKINK, Kathryn (1998), «International Norm Dynamics and Political Change », International Organization 52(4), pp. 887917.

HÜBNER, Kurt (2011), « Canada and the EU : Shaping transatlantic relations in the twenty-first century », in Europe, Canada and the Comprehensive Economic and Trade Agreement, Kurt Hübner, pp.1-17. New York: Routledge.

LEBLOND, Patrick (2010), "Canada, the European Union, and transatlantic financial governance », International Journal 66(1), pp. 57-72. 


\section{Nassiba IDEBDOU et Sébastien LABRECQUE}

--- (2014), «CETA and Financial Services", presentation at the 2014 Conference on the Canada-European Union Comprehensive Economic and Trade Agreement (CETA), McGill University, Montréal, Oct. 31-Nov. 1. http://www.mcgill.ca/fortier-chair/files/fortierchair/2014_ceta_leblond_patrick.pptx.

LÉTOURNEAU, Hugues and HEIDRICH, Pablo (2010), «Canadian Banks Abroad. Expansion and Exposure to the 2008-2009 Financial Crisis », The North-South Institute, pp. 1-44.

MEESTER, Bart De (2010), "The Global Financial Crisis and Government Support for Banks: What Role for the GATS?», Journal of International Economic Law 13(1), pp. 27-63.

Organisation Mondiale du Commerce, OMC (2015), "Annexe 1B: Accord Général sur le Commerce des Services », page consultée le 12 avril 2015, https://www.wto.org/french/docs f/legal f/26-gats 02 f.htm.

SNL Financial (2014), « SNL Financial ranks the top 20 European banks by market capitalization », SNL Financial, http://ofccolo.snl.com/cache/24786002.pdf.

The Canadian Trade Commissioner Service Foreign Affairs (2015), «Focus on the European Union », modifié le 13 mars 2015.

http://www.tradecommissioner.gc.ca/eng/document.jsp?did=97445.

WOOLCOCK, Stephen B. (2011), «European Union trade policy: The Canada-EU Comprehensive Economic and Trade Agreement (CETA) towards a new generation of FTAs? ", dans Europe, Canada and the Comprehensive Economic and Trade Agreement, Kurt Hübner, 21-40. New York: Routledge.

YOKOI-ARAI, Mamiko (2008), "GATS' Prudential Carve Out in Financial Services and its Relation with Prudential Regulation", International and Comparative Law Quarterly 57, pp. 613-648. 